\title{
HDAC8 regulates canonical Wnt pathway to promote differentiation in skeletal muscles
}

\begin{tabular}{|c|c|}
\hline Journal: & Journal of Cellular Physiology \\
\hline Manuscript ID & Draft \\
\hline Wiley - Manuscript type: & Original Research Article \\
\hline Date Submitted by the Author: & $\mathrm{n} / \mathrm{a}$ \\
\hline Complete List of Authors: & $\begin{array}{l}\text { Ferrari, Luca; Università degli Studi di Milano, Dipartimento di } \\
\text { Biotecnologie Mediche e Medicina Traslazionale } \\
\text { Bragato, Cinzia; Fondazione IRCCS Istituto Neurologico C. Besta, Milano, } \\
\text { Italy; PhD program in Neuroscience, University of Milano-Bicocca } \\
\text { Brioschi, Loredana; Università degli Studi di Milano, Dipartimento di } \\
\text { Biotecnologie Mediche e Medicina Traslazionale } \\
\text { Spreafico, Marco; Università degli Studi di Milano, Dipartimento di } \\
\text { Biotecnologie Mediche e Medicina Traslazionale } \\
\text { Esposito, Simona; Università degli Studi di Milano, Dipartimento di } \\
\text { Biotecnologie Mediche e Medicina Traslazionale } \\
\text { Pezzotta, Alex; Università degli Studi di Milano, Dipartimento di } \\
\text { Biotecnologie Mediche e Medicina Traslazionale } \\
\text { Pizzetti, Fabrizio; Department of Experimental, Diagnostic and Specialty } \\
\text { Medicine, University of Bologna, Italy. } \\
\text { Moreno Fortuny, Artal; Division of Cell Matrix Biology \& Regenerative } \\
\text { Medicine, FBMH, University of Manchester. UK.; Developmental Genetics, } \\
\text { Department of Biomedicine, University of Basel, Basel, Switzerland. } \\
\text { Giordano, Antonio; Temple University-Sbarro Institute for Cancer Research } \\
\text { and Molcular Medicine, Biology } \\
\text { Bellipanni, Gianfranco; College of Science and Technology Temple } \\
\text { University, Sbarro Institute for Cancer Research and Molecular Medicine ; } \\
\text { College of Science and Technology Temple University, Department of } \\
\text { Biology } \\
\text { Riva, Paola; Università degli Studi di Milano, Dipartimento di Biotecnologie } \\
\text { Mediche e Medicina Traslazionale } \\
\text { Frabetti, Flavia; Department of Experimental, Diagnostic and Specialty } \\
\text { Medicine, University of Bologna, Italy. } \\
\text { Viani, Paola; Università degli Studi di Milano, Dipartimento di Biotecnologie } \\
\text { Mediche e Medicina Traslazionale } \\
\text { Cossu, Giulio; Division of Cell Matrix Biology \& Regenerative Medicine, } \\
\text { FBMH, University of Manchester. UK. } \\
\text { Mora, Marina; Fondazione IRCCS Istituto Neurologico C. Besta, Milano, } \\
\text { Italy } \\
\text { Marozzi, Anna; Università degli Studi di Milano, Dipartimento di } \\
\text { Biotecnologie Mediche e Medicina Traslazionale } \\
\text { Pistocchi, Anna; University of Milan, Medical Biotechnologies and } \\
\text { Translational Medicine; University of Milan, Biosciences }\end{array}$ \\
\hline
\end{tabular}


1

2

3

4

5

6

7

8

9

10

11

12

13

14

15

16

17

18

19

20

21

22

23

24

25

26

27

28

29

30

31

32

33

34

35

36

37

38

39

40

41

42

43

44

45

46

47

48

49

50

51

52

53

54

55

56

57

58

59

60
Key Words: HDAC8, zebrafish, WNT/beta catenin, skeletal muscle

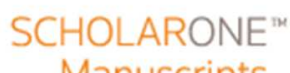

Manuscripts

John Wiley \& Sons, Inc. 


\section{HDAC8 regulates canonical Wnt pathway to promote differentiation in skeletal muscles}

2 Luca Ferrari $^{1}$, Cinzia Bragato $^{2,5}$, Loredana Brioschi $^{1 *}$, Marco Spreafico ${ }^{1}$, Simona Esposito $^{1}$, Alex 3 Pezzotta $^{1}$, Fabrizio Pizzetti ${ }^{3}$, Artal Moreno-Fortuny ${ }^{4,6}$, Gianfranco Bellipanni ${ }^{7,8}$, Antonio 4 Giordano $^{7,8}$, Paola Riva ${ }^{1}$, Flavia Frabetti ${ }^{3}$, Paola Viani ${ }^{1}$, Giulio Cossu ${ }^{4}$, Marina Mora ${ }^{2}$, Anna 5 Marozzi $^{1}$, Anna Pistocchi ${ }^{1 \#}$

6

$7{ }^{1}$ Dipartimento di Biotecnologie Mediche e Medicina Traslazionale, Università degli Studi di 8 Milano, Italy.

$9 \quad{ }^{2}$ Fondazione IRCCS Istituto Neurologico C. Besta, Milano, Italy.

$10{ }^{3}$ Department of Experimental, Diagnostic and Specialty Medicine, University of Bologna, Italy.

$11{ }^{4}$ Division of Cell Matrix Biology \& Regenerative Medicine, FBMH, University of Manchester. 12 UK.

$13{ }^{5} \mathrm{PhD}$ program in Neuroscience, University of Milano-Bicocca.

$14{ }^{6}$ Developmental Genetics, Department of Biomedicine, University of Basel, Basel, Switzerland.

$15{ }^{7}$ Department of Biology, College of Science and Technology, Temple University, Philadelphia, 16 Pennsylvania.

$17{ }^{8}$ Sbarro Institute for Cancer Research and Molecular Medicine, College of Science and Technology, 18 Temple University, Philadelphia, Pennsylvania.

$20 *$ The authors equally contributed to this work

21 \# Correspondence to: anna.pistocchi@unimi.it 
1 Running Title: HDAC8 role in skeletal muscle differentiation

2

3 Acknowledgements

4 We thank P.L. Lollini, University of Bologna, for providing rhabdomyosarcoma cell lines; Cotelli

5 F. and Mazzola M., University of Milan, for the priceless advices, practical help and useful

6 discussion of the zebrafish data. This work was supported by the AIRC, Associazione Italiana per la

7 Ricerca sul Cancro (MFAG\#18714). The funders had no role in study design, data collection and

8 interpretation, or the decision to submit the work for publication.

9

10 Keywords: HDAC8, skeletal muscle, rhabdomyosarcoma, Wnt, zebrafish 


\section{Abstract}

2 Histone deacetylase 8 (HDAC8) is a class 1 histone deacetylase and a member of the cohesin

3 complex. HDAC8 is expressed in smooth muscles but its expression in skeletal muscle has not been

4 described. We show for the first time that HDAC8 is expressed in human and zebrafish skeletal

5 muscles. Using $\mathrm{RD} / 12$ and $\mathrm{RD} / 18$ rhabdomyosarcoma cells with low and high differentiation

6 potency respectively, we highlight a specific correlation with HDAC8 expression and an advanced

7 stage of muscle differentiation. We inhibit HDAC8 activity trough the specific PCI-34051 inhibitor

8 in murine $\mathrm{C} 2 \mathrm{C} 12$ myoblasts and zebrafish embryos and we observed skeletal muscles

9 differentiation impairment. We also found a positive regulation of the canonical Wnt signalling by

10 HDAC8 that might explain muscle differentiation defects. These findings suggest a novel

11 mechanism through which HDAC8 expression in a specific time window of skeletal muscle

12 development positively regulates canonical Wnt pathway that is necessary for muscle 13 differentiation. 


\section{Introduction}

2 Skeletal muscle is necessary to accomplish fundamental functions such as the maintenance of the 3 body structure, motility and metabolism by storing and consuming energy. Skeletal muscle

4 development is a multistep process in which myogenic cells are committed to proliferating 5 myogenic precursors that then differentiate into myoblasts and myocytes that fuse to form a 6 multinucleated myotube. Several signals are essential for the regulation of skeletal muscle 7 differentiation involving transcription factors, signalling molecules, transduction pathways and 8 epigenetic modifications. Among these, the histone deacetylases (HDACs) are frequently part of the 9 regulatory elements of muscle genes (Sincennes, Brun, \& Rudnicki, n.d.). The HDAC family comprises at least 18 different enzymes classified in four classes in mammals, and has been originally identified for histone deacetylation activity and nucleosome stability. Recent evidence pinpoints their role in deacetylation also of non-histone targets such as p53 and alpha-tubulin (de Leval et al., 2006) as well as in gene transcription (Grunstein, 1997; Megee, Morgan, Mittman, \& Smith, 1990). Skeletal muscle is necessary to accomplish fundamental functions such as the maintenance of the body structure, motility and metabolism by storing and consuming energy. Skeletal muscle development is a multistep process in which myogenic cells are committed to proliferating myogenic precursors that then differentiate into myoblasts and myocytes that fuse to form a multinucleated myotube. Several signals are essential for the regulation of skeletal muscle differentiation involving transcription factors, signalling molecules, transduction pathways and epigenetic modifications. Among these, the histone deacetylases (HDACs) are frequently part of the regulatory elements of muscle genes (Sincennes, Brun, \& Rudnicki, 2016). The HDAC family comprises at least 18 different enzymes classified in four classes in mammals, and has been originally identified for histone deacetylation activity and nucleosome stability. Recent evidence pinpoints their role in deacetylation also of non-histone targets such as p53 and alpha-tubulin (de Leval et al., 2006) as well as in gene transcription (Grunstein, 1997; Megee et al., 1990). 
1 HDAC8 is the last cloned and characterized member of class I HDACs (Buggy et al., 2000; Van

2 den Wyngaert et al., 2000), it diverges from other class I enzymes as the C-terminal protein-binding

3 domain is not present, probably indicating a functional specialization during evolution (Gregoretti,

4 Lee, \& Goodson, 2004)'(Somoza et al., 2004). HDAC8 is ubiquitously expressed and can localize to

5 either the nucleus or the cytoplasm interacting with non-histone proteins such as the cohesin protein

6 SMC3, estrogen receptor a (ERRa), p53, inv(16) fusion protein (Deardorff et al., 2012; Durst,

7 Lutterbach, Kummalue, Friedman, \& Hiebert, 2003; Wilson, Tremblay, Deblois, Sylvain-Drolet, \&

8 Giguère, 2010; Wu et al., 2013). Moreover, in normal human tissues HDAC8 is expressed by

9 smooth muscle including vascular and visceral smooth muscle cells, myoepithelial cells, and 10 myofibroblasts (Durst et al., 2003; Wu et al., 2013) where interacts with cortical actin-binding 11 protein cortactin and Smooth Muscle Actin (SMA) and regulates smooth muscle contraction 12 (Buggy et al., 2000; J. Li et al., 2014; Olson et al., 2014).

13 In this study, we describe for the first time a specific HDAC8 expression in human and zebrafish 14 (Danio rerio) skeletal muscle and murine and human myogenic cells. In particular, we have 15 analyzed the time course of $H D A C 8$ expression during skeletal muscle differentiation in murine $16 \mathrm{C} 2 \mathrm{C} 12$ myoblasts and zebrafish. We noticed that $H D A C 8$ is mainly expressed when differentiation 17 is already started; moreover, in rhabdomyosarcoma derived cell lines RD/12 and RD/18 with low 18 and high differentiation potentcy respectively, the increment of $H D A C 8$ expression during the 19 differentiation is prominent in $\mathrm{RD} / 18$ than in $\mathrm{RD} / 12$ cell line. We also demonstrate that HDAC8 20 promotes muscle differentiation in vitro and in vivo as the pharmacological block of its deacetylase 21 activity inhibits myogenesis in the $\mathrm{C} 2 \mathrm{C} 12$ cellular model and in zebrafish. This function is 22 accomplished through the canonical Wnt pathway that is down-regulated when HDAC8 activity is 23 inhibited. Our results link for the first time the HDAC8 activity to broad aspects of skeletal muscle 24 development and open new possibility in the use of HDAC8 specific inhibitors (i.e. PCI-34051) 25 (Balasubramanian et al., 2008)) for therapeutic intervention on skeletal muscle diseases. 


\section{Material and Methods}

2

3

\section{Animals}

Zebrafish (Danio rerio) embryos were raised and maintained under standard conditions and national guidelines (Italian decree 4th March 2014, n.26). All experimental procedures were approved by IACUC (Institutional Animal Care and Use Committee). Zebrafish AB strains obtained from the

Wilson lab, University College London, London, United Kingdom were maintained at $28^{\circ} \mathrm{C}$ on a 14

h light/10 h dark cycle. Embryos were collected by natural spawning, staged according to Kimmel and colleagues (Kimmel, Ballard, Kimmel, Ullmann, \& Schilling, 1995) and raised at $28^{\circ} \mathrm{C}$ in fish water (Instant Ocean, 0,1\% Methylene Blue in Petri dishes), according to established techniques. We express the embryonic ages in hours post fertilization (hpf) and days post fertilization (dpf). After $24 \mathrm{hpf}$, to prevent pigmentation 0,003\% 1-phenyl-2-thiourea (Sigma-Aldrich, Saint Louis, Missouri, USA) was added to the fish water. Embryos were washed, dechorionated and anaesthetized, with $0.016 \%$ tricaine (Ethyl 3-aminobenzoate methanesulfonate salt; Sigma-Aldrich), before observations and picture acquisitions. Embryos were fixed overnight in $4 \%$ paraformaldehyde (Sigma-Aldrich) in $\mathrm{PBS}$ at $4{ }^{\circ} \mathrm{C}$, then dehydrated stepwise to methanol and stored at $-20{ }^{\circ} \mathrm{C}$.

\section{C2C12 and rhabdomyosarcoma cells}

C2C12 cells were maintained in growth medium Dulbecco's modified Eagle medium (DMEM) supplemented with $10 \%$ fetal bovine serum (FBS, Euroclone, Pero, Italy), $100 \mathrm{IU} / \mathrm{mL}$ penicillin and $100 \mu \mathrm{g} / \mathrm{mL}$ streptomycin in a humidified incubator at $37{ }^{\circ} \mathrm{C}$ with $5 \% \mathrm{CO}_{2}$. After reaching $80-90 \%$ confluence, cells were washed in phosphate-buffered saline (PBS) and differentiated in DMEM medium with Horse Serum 2\% (HS, Thermo Fisher Scientific, Waltham, MS, USA). The medium was changed every 48 hours and cultured up to 9 days of differentiation. 
$1 \mathrm{RD} / 12$ and RD/18 cell lines were two different clone originally isolated from the human embryonal

2 rhabdomyosarcoma cell lines RD by Lollini and colleagues (Lollini et al., 1991). Cells were

3 cultured in DMEM supplemented with $100 \mathrm{IU} / \mathrm{mL}$ penicillin, $100 \mu \mathrm{g} / \mathrm{mL}$ streptomycin and either

$4 \quad 10 \%$ fetal bovine serum or $2 \%$ horse serum. The culture medium was renewed every 48-72 hours up

5 to 11 days of culture in differentiation medium.

6

7

8

9

10

11

12

13

14

15

16

17

18

19

20

21

22

23

24

25

26

\section{RT-PCR and quantitative real time PCR (qPCR)}

Total RNAs were isolated from $\mathrm{C} 2 \mathrm{C} 12, \mathrm{RD} / 12, \mathrm{RD} / 18$ cells and zebrafish embryos at different developmental stages using Trizol reagent (Life Technologies, Carlsbad, CA, USA) according to the producer's instructions. After treatment with DNase I RNase-free (Roche, Basel, Switzerland) to avoid possible genomic contamination, $1 \mu \mathrm{g}$ of RNA was reverse-transcribed using the "ImPromII TM Reverse Transcription System” (Promega, Madison, WI, USA) and a mixture of oligo(dT) and random primers according to manufacturer's instructions. qPCRs on $\mathrm{C} 2 \mathrm{C} 12$ and rhabdomyosarcoma RNAs were carried out in a total volume of $20 \mu$ l containing 1 X SsoAdv Universal SYBR Green Super Mix (Bio-Rad, Hercules, CA, USA), using proper amount of the RT reaction. qPCRs were performed using the CFX-96 TM (Bio-Rad). Relative expression of HDAC8 was normalized with different reference genes, in particular TATA-box binding protein (TBP) and glyceraldehyde-3-phosphate dehydrogenase (GAPDH) was used for $\mathrm{C} 2 \mathrm{C} 12$ cell line while Actin and beta-2-microglobulin (B2M) for rhabdomyosacoma cell lines.

qPCRs in zebrafish were carried out in a total volume of $20 \mu 1$ containing $1 X$ iQ SYBR Green Super Mix (Promega), using proper amount of the RT reaction. PCRs were performed using the BioRad iCycler iQ Real Time Detection System (BioRad). For normalization purposes, rpl8 expression levels were tested in parallel with the gene of interest. Primer list in Supplementary Table 1.

In situ hybridization, histological analysis and immunohistochemistry 
1 Whole mount in situ hybridization (WISH) experiments, were carried out as described by Thisse 2 and colleagues (Thisse \& Thisse, 2008). Antisense riboprobes were previously in vitro labelled with 3 modified nucleotides (i.e. digoxigenin, fluorescein, Roche). $h d a c 8$ probe was cloned in our 4 laboratory. Primer list in Supplementary Table 1. WISH experiments were done at least in 3 batches

5 of embryos of (minimum 30 embryos for each category). Immunohistochemistry analysis was

6 carried out on 6 um-thick cryosections from human skeletal muscle biopsy. The muscle biopsy was

7 performed after informed consent, snap-frozen in isopentane/liquid nitrogen, and maintained in

8 liquid nitrogen. Cryosections were permeabilized in cold methanol (MetOH) 50\% for 1 minute and

9 MetOH 100\% for 1 minute. Cryosections were hydrated with PBS and then blocked for 30 min at room temperature in Normal Goat Serum (NGS) 1X and incubated with primary and secondary antibodies. Primary antibodies were anti-HDAC8 (1:100) (polyclonal clone (H-145): sc-11405, Santa Cruz Biotechnology, Inc., Santa Cruz, CA, USA) and anti-Lamin B (1:100) (monoclonal clone, Novocastra/YLEM, New Castle-upon-Tyne, UK). Secondary antibody were Alexa 488conjugated goat anti-mouse IgG or Alexa 546-conjugated goat anti-rabbit IgG, (Invitrogen Life Technologies, Carlsbad, CA, USA) both diluted 1:2000. As control, sections were incubated either with isotype specific IgG or the primary antibody was omitted. Sections were examined either under a Zeiss fluorescence microscope. Immunohistochemistry in zebrafish was carried out as described in Pistocchi and colleagues (Pistocchi, Gaudenzi, et al., 2013). Primary antibody was mouse anti-sarcomeric (MF20, DSHB, diluition 1:4). Secondary antibody was EnVision+ SystemHRP Labelled Polymer anti-mouse (Dako, Glostrup, Denmark). Images of embryos and sections were acquired using a microscope equipped with a digital camera with LAS Leica imaging software (Leica, Wetzlar, Germany). Images were processed using the Adobe Photoshop software and when necessary, different focal images planes of the same image have been took separately and later merged in a single image.

\section{Injections}


1 Injections were carried out on 1- to 2-cell stage embryos; the dye tracer rhodamine dextran was also 2 co-injected. To repress $h d a c 8$ mRNA translations, one morpholino was synthesized (Gene Tools

3 LLC, Philomath OR, USA) targeting hdac8-ATG. and used at the concentration of 1 pmole/embryo 4 in 1x Danieau buffer ( $\mathrm{pH} \mathrm{7,6).} \mathrm{A} \mathrm{standard} \mathrm{control} \mathrm{morpholino} \mathrm{oligonucleotide} \mathrm{(ctrl-MO)} \mathrm{was}$ 5 injected in parallel (Nasevicius \& Ekker, 2000). ATG-hdac8-MO: 5'6 CATTACTGTCGCTTTTTTCACTCAT-3’.

7

8

\section{PCI-34051 treatment}

For C2C12 cells, HDAC8 inhibitor PCI-34051 (PCI) (Cayman Chemical; Ann Arbor, MI, USA) was administrated at $25 \mu \mathrm{M}$ together with differentiating-medium; negative controls were treated with the solvent Dimethyl-sulfoxide (DMSO). The PCI was changed every 24 hours until myogenic differentiation. Zebrafish embryos after the shield developmental stage (6 hpf), were treated with $150 \mu \mathrm{M}$ PCI added to the fish water at $28^{\circ} \mathrm{C}$ kept in dark. As a control DMSO was used at the same concentration. The PCI was changed every 24 hours and the embryos are let grown until the desired developmental stage. For dose-dependent assays in zebrafish, the PCI was administrated at 50, 100, $150,250 \mu \mathrm{M}$

\section{Western Blotting}

Whole cell extracts from at least 30 zebrafish embryos were classically prepared in RIPA buffer (50 $\mathrm{mM}$ Tris- $\mathrm{HCl} \mathrm{pH}$ 7.4, 1\% NP-40, $150 \mathrm{mM} \mathrm{NaCl}, 0.25 \%$ sodium deoxycholate, 1mM EDTA, $1 \mathrm{mM}$ PMSF, protease inhibitors Roche) $(2 \mu \mathrm{l} / \mathrm{embryo}$ or $1 \mu \mathrm{l} /$ tail). Yolk was previously removed from embryos to avoid yolk protein contamination. The protein concentration was determined using a Micro BCA protein assay kit according to the manufacturer's instructions (Euroclone). $30-40 \mu \mathrm{g}$ of each sample were loaded onto a $7.5 \%$ or $10 \%$ polyacrylamide gels and subjected to electrophoresis. The proteins were then transferred onto PVDF membranes which were blocked using a blocking 
1 solution at room temperature for 1 hour prior to incubation with the primary antibodies listed in

2 Supplementary Table 2. After incubation with the HRP-conjugated secondary antibodies for $1 \mathrm{~h}$ at 3 room temperature (Secondary antibodies are listed in Supplementary Table 2). The protein bands

4 were detected using ECL detection systems. Imaging acquisition has been done with the Alliance

5 MINI HD9 AUTO Western Blot Imaging System (UVItec Limited, Cambridge) and analysed with 6 the related software (Bellipanni, Murakami, \& Weinberg, 2010). 


\section{Results}

2 HDAC8 is expressed in skeletal muscle and its expression correlates with an advance differentiated

3 state of muscle cells.

4 Several expression profiles of HDAC8 suggested that it has a ubiquitous expression in human

5 tissues, with higher expression in particular organs such as brain, pancreas, kidney, prostate, liver

6 and smooth muscles. HDAC8 transcript and protein have been detected both in the nucleus and

7 cytosol, suggesting that HDAC8 might have a variable localization within the cell, depending on the

8 cell type and its post-translational modifications such as phosphorylation (Buggy et al., 2000; de

9 Ruijter, van Gennip, Caron, Kemp, \& van Kuilenburg, 2003; Hu et al., 2000; Waltregny et al.,

10 2004). Using immunofluorescence assays we detected for the first time an expression of HDAC8 in

11 normal human skeletal muscle with a predominant nuclear localization of the protein, as shown by

12 the co-localization of HDAC8 and Lamin B (Figure 1A-C).

13 In parallel, we cloned the zebrafish orthologue of human HDAC8 (Chr 7: 51,656,099-51,710,015),

14 and by whole mount in situ hybridization analyses (WISH) we confirmed the expression of hdac8

15 in skeletal muscle of zebrafish embryos at different developmental stages (Figure 1D-F'). In

16 zebrafish the expression of $h d a c 8$ varied among the developmental stages analysed (24, 36 and 48

17 hours post fertilization, hpf), and was increased at $36 \mathrm{hpf}$ when the first myogenic wave have

18 already occurred (Stellabotte, Dobbs-McAuliffe, Fernandez, Feng, \& Devoto, 2007) (Figure 1D-F').

19 We therefore investigated a possible correlation between HDAC8 expression and skeletal muscle

20 differentiation progression. We first examined its expression in murine $\mathrm{C} 2 \mathrm{C} 12$ skeletal myogenic

21 cells, which represent a highly suitable model for analysis of myogenic differentiation. C2C12

22 myoblasts proliferate in growth medium with high serum concentration (10\% FBS) until they reach

23 confluence, while differentiation into multinucleated myotubes is triggered shifting to

24 differentiation medium with low serum concentration ( $2 \%$ horse serum). Hdac8 transcript, analysed

25 by qRT-PCR techniques, was present in C2C12 cells in growth medium and in differentiation

26 medium at 1 days but its expression was significantly increased at 7 and 9 days of differentiation 
1 (Figure 1G). In zebrafish the expression of $h d a c 8$ analysed by qRT-PCR techniques confirmed the

2 results previously shown by WISH, as the transcript is increased after the first myogenic wave when

3 differentiation was accomplished with an expression peak at $36 \mathrm{hpf}$ (Figure 1H). To further confirm

4 the correlation between HDAC8 expression and an advanced stage of differentiation, we choose two

5 different subclones of the rhabdomyosarcoma cell line RD which differ in the differentiation

6 potency: the $\mathrm{RD} / 18$ cells are able to reach a terminal differentiation while the RD/12 cells do not

7 fully differentiate (Lollini et al., 1991). The expression of HDAC8 was significantly increased at 11

8 days of differentiation with an increment of 4 fold in RD/18 and about 1 fold in RD/12 (Figure 1I).

9 HDAC8 activity regulates skeletal muscle differentiation in zebrafish and C2C12 myoblasts.

To investigate a possible function of HDAC8 in differentiating skeletal muscles we took advantage of the well characterized PCI inhibitor that blocks HDAC8 deacetylase activity (Balasubramanian et al., 2008). We administrated PCI to zebrafish embryos in-vivo and C2C12 cells in-vitro. Zebrafish embryos were treated with a concentration of $150 \mu \mathrm{M}$ of PCI from the 50\% stage of epiboly, a developmental stage in which the mesodermal layer, from which skeletal muscle derives, is positioning in the gastrula. At $48 \mathrm{hpf}$, zebrafish embryos presented morphological defects in the Central Nervous System and muscles, the regions where hdac 8 transcript was more expressed as shown in Figure 1. The PCI treated embryos could be divided in three phenotypical classes based on the severity of the CNS and muscle phenotype: class I showed a phenotype comparable to the control embryos treated with the solvent DMSO, class II presented a mild phenotype and class III presented a severe phenotype (Figure 2A-D, class quantification in E). We performed a doseresponse assay demonstrating that the observed phenotypes were correlated to the doses of PCI treatment (Suppl. Figure S1). The sarcomeric myosins, that are expressed in differentiated and functional muscle, were diminished in PCI-treated embryos in comparison to controls analysed by immunohistochemistry and Western blot techniques (Figure 2 F-I). Interestingly, same morphological defects and myosin reduction were obtained in zebrafish embryos injected with the 
1 hdac8 morpholino (hdac8-MO) that blocks Hdac8 protein production. These data indicate that the

2 skeletal muscle differentiation impairment was specific due to Hdac8 loss-of-function (Suppl.

3 Figure S2). Moreover, at 24 hpf the embryos treated with PCI did not present myogenic impairment

4 confirming that Hdac8 activity is not necessary during early skeletal muscle differentiation (Suppl.

5 Figure S3).

6 Also in-vitro, PCI treatment blocked differentiation of $\mathrm{C} 2 \mathrm{C} 12$ myoblasts in comparison to DMSO

7 treated cells. Under differentiating conditions, wild-type C2C12 cells fused into multinucleated

8 myotubes. By contrast, when challenged to differentiate in low-serum medium in presence of PCI,

$9 \mathrm{C} 2 \mathrm{C} 12$ cells remained mononucleated and maintained an undifferentiated phenotype. We assessed

10 that the differentiation of PCI treated cells was impaired in comparison to DMSO treated cells as

11 the levels of sarcomeric myosins analysed by Western blot technique were diminished (Figure 2J-

$12 \mathrm{~K}$ ).

13

14

15

HDAC8 regulates skeletal muscle differentiation through the activation of the canonical Wnt pathway.

In order to gain mechanistic insights into how HDAC8 regulates skeletal muscle differentiation, we hypothesized that it modulates the canonical Wnt pathway, a well-known regulator of skeletal muscle development and differentiation (Rudnicki \& Williams, 2015). Indeed, in a hepatocellular model, it has been demonstrated that HDAC8 positively regulates the $\beta$-catenin/TCF signalling acting in concert with EZH2 to epigenetically repress Wnt antagonists (Tian et al., 2015). Therefore, we analysed the activation status of the canonical Wnt pathway in zebrafish embryos and C2C12 myoblasts treated with PCI. The phosphorylated and active form of $\beta$-catenin was diminished by Western blot analyses in PCI treated zebrafish embryos in comparison to controls treated with the DMSO. By contrast, the levels of total $\beta$-catenin were even increased (Figure 3A, quantification in B and C). This last result is not surprising since we have seen similar up-regulation 
1 of $\beta$-Catenin in zebrafish embryos with impaired activity of the canonical Wnt pathway (Valenti et

2 al., 2015). To verify the efficiency of the PCI-mediated Hdac8 inhibition that is responsible of the

3 Wnt pathway down-regulation, we analysed the acetylation status of Smc3, a known Hdac8 target

4 (Deardorff et al., 2012). Acetylated Smc3 (Smc3ac) levels were increased following PCI treatment

5 of the embryos, confirming the block of Hdac8 activity (Figure 3D, quantification in E). Same

6 results were obtained in the $\mathrm{C} 2 \mathrm{C} 12$ cells in differentiation medium treated with PCI: Western blot

7 analyses confirmed the lower expression of active $\beta$-catenin in comparison to total $\beta$-catenin

8 (Figure Figure 3F, quantification in $\mathrm{G}$ and $\mathrm{H}$ ) and increased levels of Smc3ac following PCI

9 treatment (Figure 3I, quantification in J).

10 The Wnt pathway in zebrafish can be activated through chemical treatments such as $\mathrm{LiCl}$

11 (Pistocchi, Fazio, et al., 2013). Therefore, to further demonstrate that skeletal muscle differentiation

12 impairment observed with PCI-mediated Hdac8 inhibition was specifically due to Wnt pathway 13 down-regulation, we re-activated the pathway adding $\mathrm{LiCl}$ in PCI-treated zebrafish embryos. The 14 morphological defects presented by PCI-treated embryos at $36 \mathrm{hpf}$ (embryos with morphological 


\section{Discussion}

2 In previous works HDAC8 was shown to be expressed in smooth muscle cells in association with

3 SMA and cortactin (Jia Li et al., 2014)nd its silencing by RNA interference (RNAi) impairs the

4 contraction of smooth muscle cultured cells (Waltregny et al., 2005). However, the role and

5 mechanism of HDAC8 action in smooth muscle tissues are largely unknown. In this work, we

6 described for the first time the expression and role of HDAC8 in the skeletal muscle. Firstly, we

7 demonstrated that HDAC8 is expressed in human and zebrafish skeletal muscle; then we analysed

8 the expression of $H D A C 8$ during muscle differentiation in the murine $\mathrm{C} 2 \mathrm{C} 12$ skeletal muscle cells,

9 during zebrafish muscle development and in two types of rhabdomyosarcoma cells with various 10 degree of invasiveness correlating to their ability to differentiate (RD/12 and RD/18). We decided

11 to include these cells in the expression analyses as it has been reported that HDACi synergize with 12 current anticancer drugs to induce apoptosis in rhabdomyosarcoma although the authors observed a 13 switch to myogenic differentiation (Vleeshouwer-Neumann et al., 2015,'Di Pompo et al., 2015). 14 Interestingly, we correlate the expression of $H D A C 8$ with an advanced differentiation state of 15 skeletal muscles. Indeed, both in $\mathrm{C} 2 \mathrm{C} 12$ cells and zebrafish, $H D A C 8$ expression is weak in the 16 initial phases and increases later during the muscle differentiation process. These data are even 17 more striking in the rhabdomyosarcoma cells, where the RD/18 cell line cultured in the 18 differentiation medium for 11 days shows a greater increase in the HDAC8 expression compared to 19 the $\mathrm{RD} / 12$ cell line maintained in the same conditions. This increase correlates with the 20 differentiation capacity of the two cell lines.

21 For functional analyses, we treated the $\mathrm{C} 2 \mathrm{C} 12$ cells and the zebrafish embryos with the HDAC8 22 inhibitor PCI-34051. In zebrafish, we also performed loss-of-function studies by injecting the 23 oligonucleotide antisense morpholino designed against hdac8 to compare and confirm the results 24 obtained with the PCI-34051 treatment. Both in the cellular and zebrafish models with reduced 25 HDAC8 activity, we observed an impairment in muscle differentiation following the initial 26 myoblast commitment, in line with the kinetic of $H D A C 8$ expression previously analysed. In the 
$1 \mathrm{C} 2 \mathrm{C} 12$ cells, myoblasts were formed but failed to fuse in myotubes and to express the markers of

2 differentiation; in zebrafish, the levels of functional myosins were reduced after 24 hpf but the

3 myogenic program started, as demonstrated by the proper expression of the MRFs MyoD and Myog

4 and by the presence of myosin proteins. Interestingly, it has been already shown that the levels of

5 myogenin were not affected by myoblast exposure to HDACi (Iezzi, Cossu, Nervi, Sartorelli, \&

6 Puri, 2002), suggesting that HDACi selectively activate late muscle markers. It is also reported a

7 dual action for HDACi on muscle differentiation, depending on the stage of administration:

8 previous studies reported that HDACi have different effects by promoting or inhibiting myogenesis

9 (Steinbach, Wolffe, \& Rupp, 1997) and this discrepancy might be explained by the stage-specific effects of HDACi exposure. In zebrafish embryos, we performed the Hdac8 inhibition by adding the PCI-34051 inhibitor after the shield stage $(6 \mathrm{hpf})$ to prevent gross morphological defects in the initial phase of gastrulation when mesoderm is defined.

The block on muscle differentiation observed following HDAC8 inhibition is correlated with the down-regulation of the canonical Wnt pathway. Several works demonstrate that the formation of skeletal muscle is tightly modulated by Wnt signalling for self-renewal and muscle differentiation and its dysregulation leads to perturbation of muscle fibers. Chemical modulation of the Wnt/ $\beta$ catenin pathway in differentiating myoblasts, using the activator $\mathrm{LiCl}$, increases both the number and size of $\mathrm{C} 2 \mathrm{C} 12$ myotubes while inhibitors of $\mathrm{Wnt} / \beta$-catenin signalling result in a significant decrease in myotube length (Abraham, 2016). Indeed, the Wnt target $\beta$-catenin interacts directly with MyoD, enhancing its binding to $\mathrm{E}$ box elements and its transcriptional activity of muscle specific genes. This transactivation is inhibited when $\beta$-catenin is deficient or the interaction between MyoD and $\beta$-catenin is disrupted (Kim, Mei 2008). We demonstrate that the reduction of myosins observed in PCI-34051 treated embryos was caused by a decrease in activated $\beta$-catenin levels. A mechanism by which HDAC8 regulates the canonical Wnt pathway has been recently described in human NAFLD-associated hepatocellular carcinoma (HCC) by Tian and colleagues 
1 (Tian, Mok, Yang, \& Cheng, 2016). HDAC8 physically interacts with the polycomb protein

2 enhancer of zeste homolog 2 (EZH2) and contributes to the activation of Wnt/ $\square$-catenin signalling.

3 Further analyses are necessary to demonstrate whether this mechanism is conserved also in skeletal

4 muscle and acetylome profile following PCI inhibition may uncover HDAC8-related targets.

5 Dis-regulation of canonical Wnt signalling has been reported in different muscle pathologies, such

6 as Duchenne Muscular Dystrophy (DMD) (Trensz, Haroun, Cloutier, Richter, \& Grenier, 2010),

7 FascioScapuloHumeral Muscular Dystrophy (FSHD) (Block et al., 2013) and OculoPharyngeal

8 Muscular Dystrophy (OPMD) (Abu-Baker et al., 2013). Inhibition of canonical Wnt signalling by

9 Dkk in a mouse model for DMD (mdx), was shown to reduce fibrosis (Trensz et al., 2010). HDACi

10 are recently emerged as potential pharmacological strategies for cancer treatment, and several of

11 them are already approved by the international Drug Administration agencies. The increasing

12 interest and use of HDACi has led to the development of class-specific inhibitors, such as the PCI-

13 34051, which helps us to uncover the functional role of HDAC8 in skeletal muscle differentiation

14 and, in the future, might ameliorate the phenotype in pathological conditions. Based on the

15 numerous beneficial effects of HDACi in skeletal muscle under pathological conditions, we believe 16 that they are promising therapeutics. 


\section{Competing interests}

2 All authors declare that they have no conflict of interest. Declaration of interest: none.

3

4 Author contributions: conceived and designed the experiments: $\mathrm{AP}^{1}, \mathrm{AM}^{1}, \mathrm{PV}^{1}$. Performed the 5 experiments on human samples: $\mathrm{CB}^{2,5}, \mathrm{MM}^{2}$. Performed the experiments in zebrafish: $\mathrm{MS}^{1}, \mathrm{SE}^{1}$, $6 \mathrm{LB}^{1}, \mathrm{AP}^{1}, \mathrm{AP}^{1}, \mathrm{LF}^{1}$. Performed the experiments in $\mathrm{C} 2 \mathrm{C} 12$ cells: $\mathrm{LF}^{1}, \mathrm{AMF}^{4,6}, \mathrm{FP}^{3}, \mathrm{FF}^{3}$. Performed 7 the experiments in rhabdomyosarcoma cells: $\mathrm{FP}^{3}, \mathrm{FF}^{3}$. Analyzed the data on human sample: $\mathrm{CB}^{2,5}$, $8 \mathrm{MM}^{2}$. Analysed the data in zebrafish: $\mathrm{MS}^{1}, \mathrm{SE}^{1}, \mathrm{LB}^{1}, \mathrm{AP}^{1}, \mathrm{AP}^{1}, \mathrm{LF}^{1}, \mathrm{PR}^{1}, \mathrm{AG}^{7,8}, \mathrm{~GB}^{7,8}$. Analysed 9 the data in $\mathrm{C} 2 \mathrm{C} 12$ cells: $\mathrm{LF}^{1}, \mathrm{PR}^{1}, \mathrm{FP}^{3}, \mathrm{FF}^{3}, \mathrm{FB}, \mathrm{AMF}^{4,6}, \mathrm{GC}^{4}$. Wrote the paper: $\mathrm{AP}^{1}$. Supervised 10 paper drafting: $\mathrm{AP}^{1}, \mathrm{AG}^{7,8}, \mathrm{~GB}^{7,8}$. Supervised the research project $\mathrm{AP}^{1}$. 
1

2

3

4

5

6

7

8

9

10

11

12

\section{References}

Abraham, S. T. (2016). A role for the Wnt3a/ $\beta$-catenin signaling pathway in the myogenic program of C2C12 cells. In Vitro Cellular and Developmental Biology - Animal, 52(9), 935-941. https://doi.org/10.1007/s11626-016-0058-5

Abu-Baker, A., Laganiere, J., Gaudet, R., Rochefort, D., Brais, B., Neri, C., ... Rouleau, G. A. (2013). Lithium chloride attenuates cell death in oculopharyngeal muscular dystrophy by perturbing Wnt/ß-catenin pathway. Cell Death \& Disease, 4(10), e821. https://doi.org/10.1038/cddis.2013.342

Balasubramanian, S., Ramos, J., Luo, W., Sirisawad, M., Verner, E., \& Buggy, J. J. (2008). A novel histone deacetylase 8 (HDAC8)-specific inhibitor PCI-34051 induces apoptosis in T-cell lymphomas. Leukemia, 22(5), 1026-1034. https://doi.org/10.1038/leu.2008.9

Bellipanni, G., Murakami, T., \& Weinberg, E. S. (2010). Molecular dissection of Otx1 functional domains in the zebrafish embryo. Journal of Cellular Physiology, 222(2), 286-293. https://doi.org/10.1002/jcp.21944

Block, G. J., Narayanan, D., Amell, A. M., Petek, L. M., Davidson, K. C., Bird, T. D., ... Miller, D. G. (2013). Wnt/ $\beta$-catenin signaling suppresses DUX4 expression and prevents apoptosis of FSHD muscle cells. Human Molecular Genetics, 22(23), 4661-72. https://doi.org/10.1093/hmg/ddt314

Buggy, J. J., Sideris, M. L., Mak, P., Lorimer, D. D., McIntosh, B., \& Clark, J. M. (2000). Cloning and characterization of a novel human histone deacetylase, HDAC8. The Biochemical Journal, 350 Pt 1, 199-205. https://doi.org/10.1042/BJ3500199

de Leval, L., Waltregny, D., Boniver, J., Young, R. H., Castronovo, V., \& Oliva, E. (2006). Use of histone deacetylase 8 (HDAC8), a new marker of smooth muscle differentiation, in the classification of mesenchymal tumors of the uterus. The American Journal of Surgical Pathology, 30(3), 319-27. https://doi.org/10.1097/01.pas.0000188029.63706.31

de Ruijter, A. J. M., van Gennip, A. H., Caron, H. N., Kemp, S., \& van Kuilenburg, A. B. P. (2003). 
Histone deacetylases (HDACs): characterization of the classical HDAC family. The Biochemical Journal, 370(Pt 3), 737-49. https://doi.org/10.1042/BJ20021321

Deardorff, M. A., Bando, M., Nakato, R., Watrin, E., Itoh, T., Minamino, M., ... Shirahige, K. (2012). HDAC8 mutations in Cornelia de Lange syndrome affect the cohesin acetylation cycle. Nature, 489(7415), 313-317. https://doi.org/10.1038/nature11316

Di Pompo, G., Salerno, M., Rotili, D., Valente, S., Zwergel, C., Avnet, S., ... Mai, A. (2015). Novel Histone Deacetylase Inhibitors Induce Growth Arrest, Apoptosis, and Differentiation in Sarcoma Cancer Stem Cells. Journal of Medicinal Chemistry, 58(9), 4073-4079. https://doi.org/10.1021/acs.jmedchem.5b00126

Durst, K. L., Lutterbach, B., Kummalue, T., Friedman, A. D., \& Hiebert, S. W. (2003). The inv(16) fusion protein associates with corepressors via a smooth muscle myosin heavy-chain domain. Molecular and Cellular Biology, 23(2), 607-19. https://doi.org/10.1128/MCB.23.2.607619.2003

Gregoretti, I. V., Lee, Y. M., \& Goodson, H. V. (2004). Molecular evolution of the histone deacetylase family: Functional implications of phylogenetic analysis. Journal of Molecular Biology, 338(1), 17-31. https://doi.org/10.1016/j.jmb.2004.02.006

Grunstein, M. (1997). Histone acetylation in chromatin structure and transcription. Nature, 389(6649), 349-352. https://doi.org/10.1038/38664

Hu, E., Chen, Z., Fredrickson, T., Zhu, Y., Kirkpatrick, R., Zhang, G. F., ... Winkler, J. (2000). Cloning and characterization of a novel human class I histone deacetylase that functions as a transcription repressor. Journal of Biological Chemistry, 275(20), 15254-15264. https://doi.org/10.1074/jbc.M908988199

Iezzi, S., Cossu, G., Nervi, C., Sartorelli, V., \& Puri, P. L. (2002). Stage-specific modulation of skeletal myogenesis by inhibitors of nuclear deacetylases. Proceedings of the National Academy of Sciences of the United States of America, 99(11), 7757-62. https://doi.org/10.1073/pnas.112218599 
1 Kimmel, C., Ballard, W., Kimmel, S., Ullmann, B., \& Schilling, T. (1995). Stages of embryonic

2

3

4

5

6

7

8

9

10 development of the zebrafish. Developmental Dynamics, 203(3), 253-310. https://doi.org/10.1002/aja.1002030302

Li, J., Chen, S., Cleary, R. A., Wang, R., Gannon, O. J., Seto, E., \& Tang, D. D. (2014). Histone deacetylase 8 regulates cortactin deacetylation and contraction in smooth muscle tissues. American Journal of Physiology. Cell Physiology, 307(3), C288-95. https://doi.org/10.1152/ajpcell.00102.2014

Li, J., Chen, S., Cleary, R. A., Wang, R., Gannon, O. J., Seto, E., \& Tang, D. D. (2014). Histone deacetylase 8 regulates cortactin deacetylation and contraction in smooth muscle tissues. AJP: Cell Physiology, 307(3), C288-C295. https://doi.org/10.1152/ajpcell.00102.2014

Lollini, P. L., De Giovanni, C., Landuzzi, L., Nicoletti, G., Scotlandi, K., \& Nanni, P. (1991). Reduced metastatic ability of in vitro differentiated human rhabdomyosarcoma cells. Invasion \& Metastasis, 11(2), 116-24. Retrieved from http://www.ncbi.nlm.nih.gov/pubmed/1917385

Megee, P. C., Morgan, B. A., Mittman, B. A., \& Smith, M. M. (1990). Genetic analysis of histone H4: Essential role of lysines subject to reversible acetylation. Science, 247(4944), 841-845. https://doi.org/10.1126/science.2106160

Nasevicius, a, \& Ekker, S. C. (2000). Effective targeted gene "knockdown" in zebrafish. Nature Genetics, 26(2), 216-20. https://doi.org/10.1038/79951

Olson, D. E., Udeshi, N. D., Wolfson, N. A., Pitcairn, C. A., Sullivan, E. D., Jaffe, J. D., ... Holson, E. B. (2014). An unbiased approach to identify endogenous substrates of "histone" deacetylase 8. ACS Chemical Biology, 9(10), 2210-2216. https://doi.org/10.1021/cb500492r

Pistocchi, A., Fazio, G., Cereda, A., Ferrari, L., Bettini, L. R., Messina, G., ... Massa, V. (2013). Cornelia de Lange Syndrome: NIPBL haploinsufficiency downregulates canonical Wnt pathway in zebrafish embryos and patients fibroblasts. Cell Death and Disease, 4(10), e866. https://doi.org/10.1038/cddis.2013.371

Pistocchi, A., Gaudenzi, G., Foglia, E., Monteverde, S., Moreno-Fortuny, A., Pianca, A., ... 
Messina, G. (2013). Conserved and divergent functions of Nfix in skeletal muscle development during vertebrate evolution. Development (Cambridge), 140(7). https://doi.org/10.1242/dev.076315

Rudnicki, M. A., \& Williams, B. O. (2015). Wnt signaling in bone and muscle. Bone. https://doi.org/10.1016/j.bone.2015.02.009

Sincennes, M.-C., Brun, C. E., \& Rudnicki, M. A. (n.d.). Tissue-Specific Progenitor and Stem Cells Concise Review: Epigenetic Regulation of Myogenesis in Health and Disease. https://doi.org/10.5966/sctm.2015-0266

Sincennes, M.-C., Brun, C. E., \& Rudnicki, M. A. (2016). Concise Review: Epigenetic Regulation of Myogenesis in Health and Disease. STEM CELLS Translational Medicine, 5(3), 282-290. https://doi.org/10.5966/sctm.2015-0266

Somoza, J. R., Skene, R. J., Katz, B. A., Mol, C., Ho, J. D., Jennings, A. J., .. Tari, L. W. (2004). Structural snapshots of human HDAC8 provide insights into the class I histone deacetylases. Structure, 12(7), 1325-1334. https://doi.org/10.1016/j.str.2004.04.012

Steinbach, O. C., Wolffe, A. P., \& Rupp, R. A. W. (1997). Somatic linker histones cause loss of mesodermal competence in Xenopus. Nature, 389(6649), 395-399. https://doi.org/10.1038/38755

Stellabotte, F., Dobbs-McAuliffe, B., Fernandez, D. A., Feng, X., \& Devoto, S. H. (2007). Dynamic somite cell rearrangements lead to distinct waves of myotome growth. Development, 134(7), 1253-1257. https://doi.org/10.1242/dev.000067

Thisse, C., \& Thisse, B. (2008). High-resolution in situ hybridization to whole-mount zebrafish embryos. Nature Protocols, 3(1), 59-69. https://doi.org/10.1038/nprot.2007.514

Tian, Y., Mok, M., Yang, P., \& Cheng, A. (2016). Epigenetic Activation of Wnt//-Catenin Signaling in NAFLD-Associated Hepatocarcinogenesis. Cancers, 8(8), 76. https://doi.org/10.3390/cancers8080076

Tian, Y., Wong, V. W. S., Wong, G. L. H., Yang, W., Sun, H., Shen, J., ... Chan, H. L. Y. (2015). 
Histone deacetylase HDAC8 promotes insulin resistance and $\beta$-catenin activation in NAFLDassociated hepatocellular carcinoma. Cancer Research, 75(22), 4803-4816. https://doi.org/10.1158/0008-5472.CAN-14-3786

Trensz, F., Haroun, S., Cloutier, A., Richter, M. V., \& Grenier, G. (2010). A muscle resident cell population promotes fibrosis in hindlimb skeletal muscles of mdx mice through the Wnt canonical pathway. American Journal of Physiology-Cell Physiology, 299(5), C939-C947. https://doi.org/10.1152/ajpcell.00253.2010

Valenti, F., Ibetti, J., Komiya, Y., Baxter, M., Lucchese, A. M., Derstine, L., ... Bellipanni, G. (2015). The Increase in Maternal Expression of axin1 and axin2 Contribute to the Zebrafish Mutant Ichabod Ventralized Phenotype. Journal of Cellular Biochemistry, 116(3), 418-430. https://doi.org/10.1002/jcb.24993

Van den Wyngaert, I., de Vries, W., Kremer, a, Neefs, J., Verhasselt, P., Luyten, W. H., \& Kass, S. U. (2000). Cloning and characterization of human histone deacetylase 8. FEBS Letters, 478(12), 77-83. https://doi.org/10.1016/S0014-5793(00)01813-5

Vleeshouwer-Neumann, T., Phelps, M., Bammler, T. K., MacDonald, J. W., Jenkins, I., \& Chen, E. Y. (2015). Histone Deacetylase Inhibitors Antagonize Distinct Pathways to Suppress Tumorigenesis of Embryonal Rhabdomyosarcoma. PloS One, 10(12), e0144320. https://doi.org/10.1371/journal.pone.0144320

Waltregny, D., De Leval, L., Glénisson, W., Ly Tran, S., North, B. J., Bellahcène, A., ... Castronovo, V. (2004). Expression of histone deacetylase 8, a class I histone deacetylase, is restricted to cells showing smooth muscle differentiation in normal human tissues. The American Journal of Pathology, 165(2), 553-564. https://doi.org/10.1016/S00029440(10)63320-2

Waltregny, D., Glénisson, W., Tran, S. L., North, B. J., Verdin, E., Colige, A., \& Castronovo, V. (2005). Histone deacetylase HDAC8 associates with smooth muscle alpha-actin and is essential for smooth muscle cell contractility. Faseb, 19(8), 966-8. 
1 https://doi.org/10.1096/fj.04-2303fje

2 Wilson, B. J., Tremblay, A. M., Deblois, G., Sylvain-Drolet, G., \& Giguère, V. (2010). An

3 acetylation switch modulates the transcriptional activity of estrogen-related receptor alpha.

$4 \quad$ Molecular Endocrinology, 24(7), 1349-1358. https://doi.org/10.1210/me.2009-0441

5 Wu, J., Du, C., Lv, Z., Ding, C., Cheng, J., Xie, H., ... Zheng, S. (2013). The up-regulation of

6 histone deacetylase 8 promotes proliferation and inhibits apoptosis in hepatocellular

7 carcinoma. Digestive Diseases and Sciences, 58(12). https://doi.org/10.1007/s10620-013-

$8 \quad 2867-7$

9

10 


\section{Figures legends}

2 Figure 1: HDAC8 is expressed in human, murine and zebrafish skeletal muscle and its 3 expression correlates with differentiation potency. $(A-C)$ HDAC8 protein expression in normal 4 human skeletal muscles. Immunofluorescence staining of HDAC8 $(A)$, Lamin B $(B)$ and merge of 5 the two signals $(C)$. The localization of HDAC8 in human skeletal muscle is predominantly nuclear 6 as shown by the co-localization with the Lamin B protein. (D-F) hdac8 mRNA expression in 7 zebrafish. WISH analyses of hdac 8 transcript localization in skeletal muscle of zebrafish embryos at $8 \quad 24 \mathrm{hpf}(D), 36 \mathrm{hpf}(E)$ and $48 \mathrm{hpf}(F)$. Transverse histological sections of the previously hybridized 9 embryos show the localization of $h d a c 8$ transcript in the myotome $\left(D^{\prime}, E^{\prime}, F^{\prime}\right) .(G) H d a c 8$ qRT-PCR 10 analyses on murine $\mathrm{C} 2 \mathrm{C} 12$ myoblasts at different stages of differentiation. Hdac 8 expression is 11 increased at 7-9 days after the induction of the differentiation when differentiation is accomplished. 12 (H) hdac8 qRT-PCR analyses on RNA from 24, 36 and 48 hpf zebrafish embryos. hdac8 expression 13 is increased at $36 \mathrm{hpf}$ when the first myogenic wave is completed. (I) HDAC8 qRT-PCR analyses 14 on $\mathrm{RD} / 12$ and $\mathrm{RD} / 18$ rhabdomyosarcoma cells. At 11 days after the induction of differentiation, $15 H D A C 8$ is more expressed in $\mathrm{RD} / 18$ cells that are able to fully differentiate in comparison to $\mathrm{RD} / 12$ 16 cells. Scale bar represents $50 \mu \mathrm{m}$ in $(A-C)$ and $100 \mu \mathrm{m}\left(D-F^{\prime}\right)$. Asterisks represent **p $<0.01$, $17 * * * \mathrm{p}<0.001$, Student's t test.

Figure 2: Inhibition of HDAC8 activity reduces skeletal muscle differentiation in zebrafish embryos and murine C2C12 myblasts. (A-E) In-vivo treatment of zebrafish embryos with DMSO or PCI. Different phenotypical classes with increased severity ( $B-D$; quantification in $E)$ with PCI treatment compared to the control embryos treated with the DMSO $(A)$. $(F-G)$ Immunohistochemical staining (IHC) and (H-I) western blot analyses of sarcomeric myosins. Sarcomeric myosins are reduced in PCI treated embryos at $48 \mathrm{hpf}(G)$ in comparison to controls $(F)$. Western blot analyses $(H$; quantification in $I)$ confirmed all MyHC reduction in PCI treated embryos in comparison to controls. $(J-K)$. Inhibition of HDAC8 activity impaired $\mathrm{C} 2 \mathrm{C} 12$ 
differentiation. Western blot analyses ( $J$; quantification in $K$ ) confirmed all MyHC reduction in PCI

2 treated $\mathrm{C} 2 \mathrm{C} 12$ cells in comparison to DMSO treated. Scale bars indicates $100 \mu \mathrm{m}$ in $(A, F)$.

3 Asterisks represent $* \mathrm{p}<0.05$, Student's $\mathrm{t}$ test.

4

Figure 3: HDAC8 activates canonical Wnt signalling. $(A-C)$ Canonical Wnt signalling was decreased with the PCI treatment in zebrafish embryos. (A) Active $\beta$-catenin was decreased in PCI treated embryos in comparison to DMSO controls while total $\beta$-catenin was increased by Western blot analyses and relative quantifications $(B-C)$. (D-E) The efficacy of PCI treatment was verified by the acetylation status of the Hdac8 target Smc3. (D) Smc3ac levels were increased in PCI treated embryos in comparison to DMSO controls, quantification in $(E)$. (F-H) Canonical Wnt signalling was decreased with the PCI treatment in $\mathrm{C} 2 \mathrm{C} 12$ cells in differentiation medium. $(F)$ Active $\beta$ catenin was decreased in PCI treated $\mathrm{C} 2 \mathrm{C} 12$ cells in comparison to those treated with DMSO, while total $\beta$-catenin was increased by Western blot analyses and relative quantifications $(G-H)$. (I-J) The efficacy of PCI treatment in the $\mathrm{C} 2 \mathrm{C} 12$ was verify by the acetylation status of the $\mathrm{Hdac} 8$ target Smc3. (I) Smc3ac levels were increased in PCI treated C2C12 in comparison to DMSO controls, quantification in $(J)$. Asterisks represent $* \mathrm{p}<0.05, * * * \mathrm{p}<0.001$, Student's t test.

Figure 4: The HDAC8-mediated positive regulation of Wnt signalling is responsible for skeletal muscle differentiation. $(A-C)$ Morphological defect presented by PCI-treated embryos were rescued by $\mathrm{LiCl}$ addition. (D-F) Skeletal muscle differentiation was rescued when the Wnt pathway was restored by $\mathrm{LiCl}$ in PCI treated zebrafish embryos. (D) Sarcomeric myosins, analysed by Western blot techniques, decreased in PCI treated embryos and returned comparable to those treated with DMSO when Wnt pathway was rescued adding $\mathrm{LiCl}$ (quantification in $E$ ). The efficacy of $\mathrm{LiCl}$ treatment was verify measuring the active $\beta$-catenin by Western blot techniques (quantification in $F$ ). Asterisks represent * $\mathrm{p}<0.05$, Student's $\mathrm{t}$ test. 
John Wiley \& Sons, Inc. 

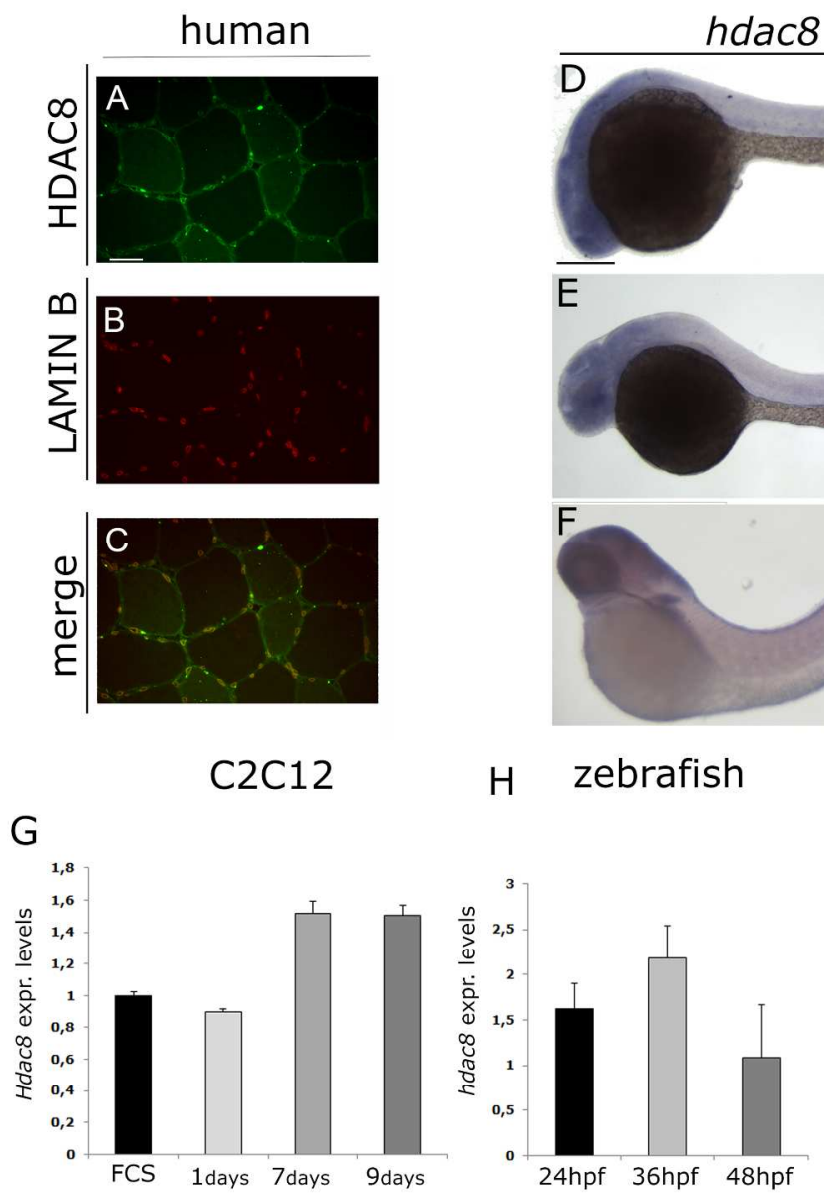

hdac8 zebrafish
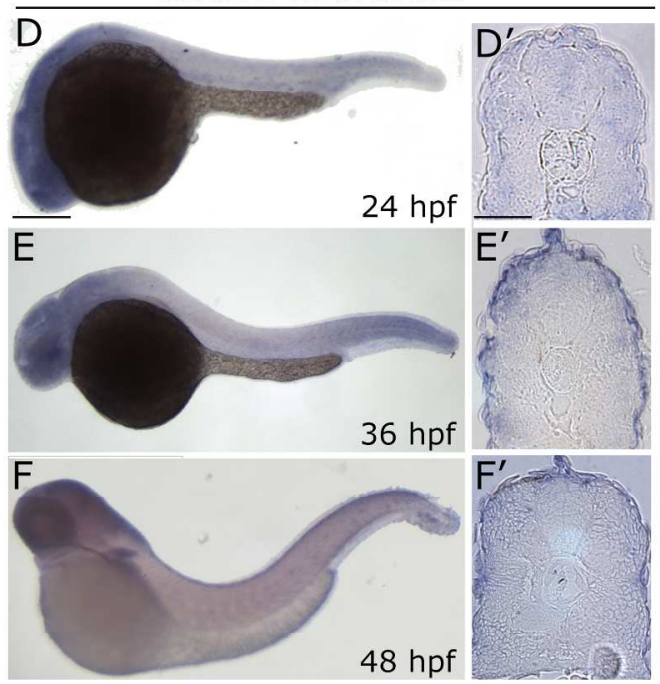

$8 \mathrm{hpf}$
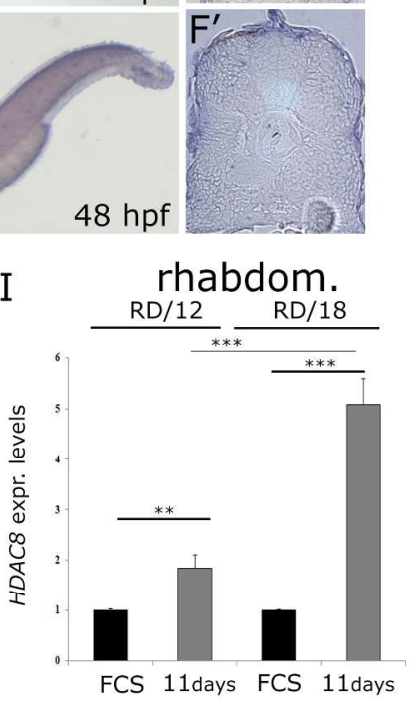

Figure 1: HDAC8 is expressed in human, murine and zebrafish skeletal muscle and its expression correlates with differentiation potency. (A-C) HDAC8 protein expression in normal human skeletal muscles.

Immunofluorescence staining of HDAC8 (A), Lamin B (B) and merge of the two signals (C). The localization of HDAC8 in human skeletal muscle is predominantly nuclear as shown by the co-localization with the Lamin B protein. (D-F) hdac8 mRNA expression in zebrafish. WISH analyses of hdac8 transcript localization in skeletal muscle of zebrafish embryos at $24 \mathrm{hpf}(\mathrm{D}), 36 \mathrm{hpf}(\mathrm{E})$ and $48 \mathrm{hpf}(\mathrm{F})$. Transverse histological sections of the previously hybridized embryos show the localization of hdac8 transcript in the myotome $\left(\mathrm{D}^{\prime}, \mathrm{E}^{\prime}, \mathrm{F}^{\prime}\right)$. (G) Hdac8 qRT-PCR analyses on murine $\mathrm{C} 2 \mathrm{C} 12$ myoblasts at different stages of differentiation. Hdac8 expression is increased at 7-9 days after the induction of the differentiation when differentiation is accomplished. (H) hdac8 qRT-PCR analyses on RNA from 24, 36 and 48 hpf zebrafish embryos. hdac8 expression is increased at $36 \mathrm{hpf}$ when the first myogenic wave is completed. (I) HDAC8 qRT-PCR analyses on $\mathrm{RD} / 12$ and $\mathrm{RD} / 18$ rhabdomyosarcoma cells. At 11 days after the induction of differentiation, HDAC8 is more expressed in $\mathrm{RD} / 18$ cells that are able to fully differentiate in comparison to RD/12 cells. Scale bar represents $50 \mu \mathrm{m}$ in $(A-C)$ and $100 \mu \mathrm{m}\left(D-F^{\prime}\right)$. Asterisks represent $* * p<0.01, * * * p<0.001$, Student's t test.

$$
216 \times 202 \mathrm{~mm} \text { ( } 300 \times 300 \text { DPI) }
$$




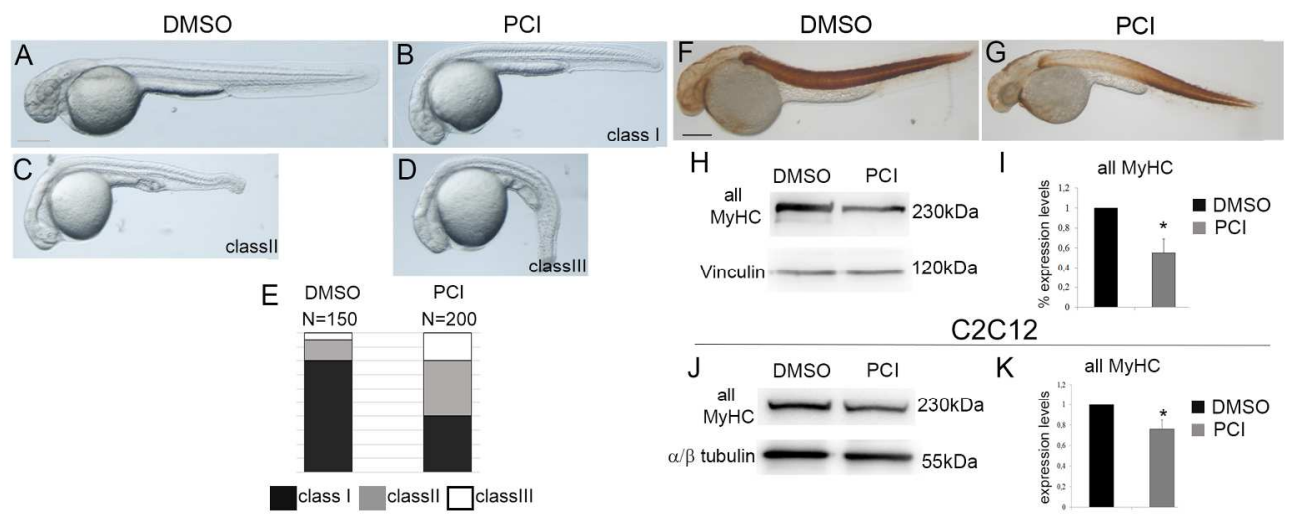

Figure 2: Inhibition of HDAC8 activity reduces skeletal muscle differentiation in zebrafish embryos and murine C2C12 myblasts. (A-E) In-vivo treatment of zebrafish embryos with DMSO or PCI. Different phenotypical classes with increased severity (B-D; quantification in E) with PCI treatment compared to the control embryos treated with the DMSO (A). (F-G) Immunohistochemical staining (IHC) and (H-I) western blot analyses of sarcomeric myosins. Sarcomeric myosins are reduced in PCI treated embryos at $48 \mathrm{hpf}(\mathrm{G})$ in comparison to controls ( $F)$. Western blot analyses ( $\mathrm{H}$; quantification in $\mathrm{I}$ ) confirmed all MyHC reduction in

PCI treated embryos in comparison to controls. (J-K). Inhibition of HDAC8 activity impaired C2C12 differentiation. Western blot analyses ( $\mathrm{J}$; quantification in $\mathrm{K}$ ) confirmed all MyHC reduction in PCI treated C2C12 cells in comparison to DMSO treated. Scale bars indicates $100 \mu \mathrm{m}$ in (A, F). Asterisks represent $* p<0.05$, Student's t test.

$588 \times 233 \mathrm{~mm}(96 \times 96$ DPI $)$ 


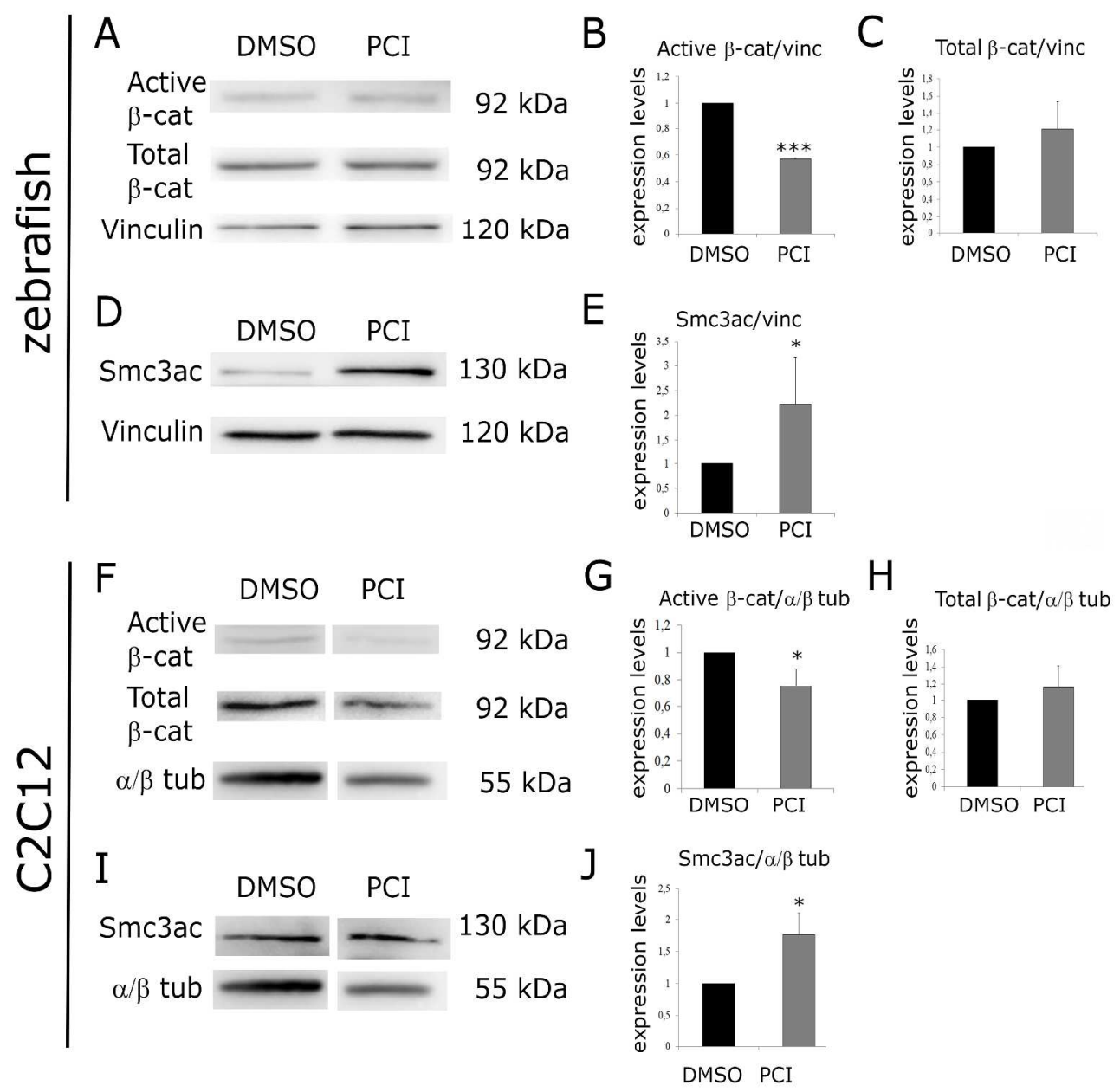

Figure 3: HDAC8 activates canonical Wnt signalling. (A-C) Canonical Wnt signalling was decreased with the

PCI treatment in zebrafish embryos. (A) Active $\beta$-catenin was decreased in PCI treated embryos in comparison to DMSO controls while total $\beta$-catenin was increased by Western blot analyses and relative quantifications (B-C). (D-E) The efficacy of PCI treatment was verified by the acetylation status of the Hdac8 target Smc3. (D) Smc3ac levels were increased in PCI treated embryos in comparison to DMSO controls, quantification in (E). (F-H) Canonical Wnt signalling was decreased with the PCI treatment in $\mathrm{C} 2 \mathrm{C} 12$ cells in differentiation medium. (F) Active $\beta$-catenin was decreased in PCI treated $\mathrm{C} 2 \mathrm{C} 12$ cells in comparison to those treated with DMSO, while total $\beta$-catenin was increased by Western blot analyses and relative quantifications (G-H). (I-J) The efficacy of PCI treatment in the C2C12 was verify by the acetylation status of the Hdac8 target Smc3. (I) Smc3ac levels were increased in PCI treated C2C12 in comparison to DMSO controls, quantification in (J). Asterisks represent $* \mathrm{p}<0.05, * * * \mathrm{p}<0.001$, Student's t test. 
PCI

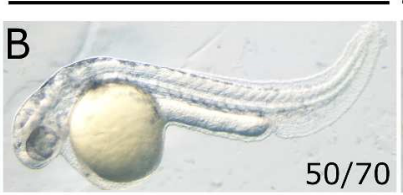

\section{$\mathrm{PCI}+\mathrm{LiCl}$}
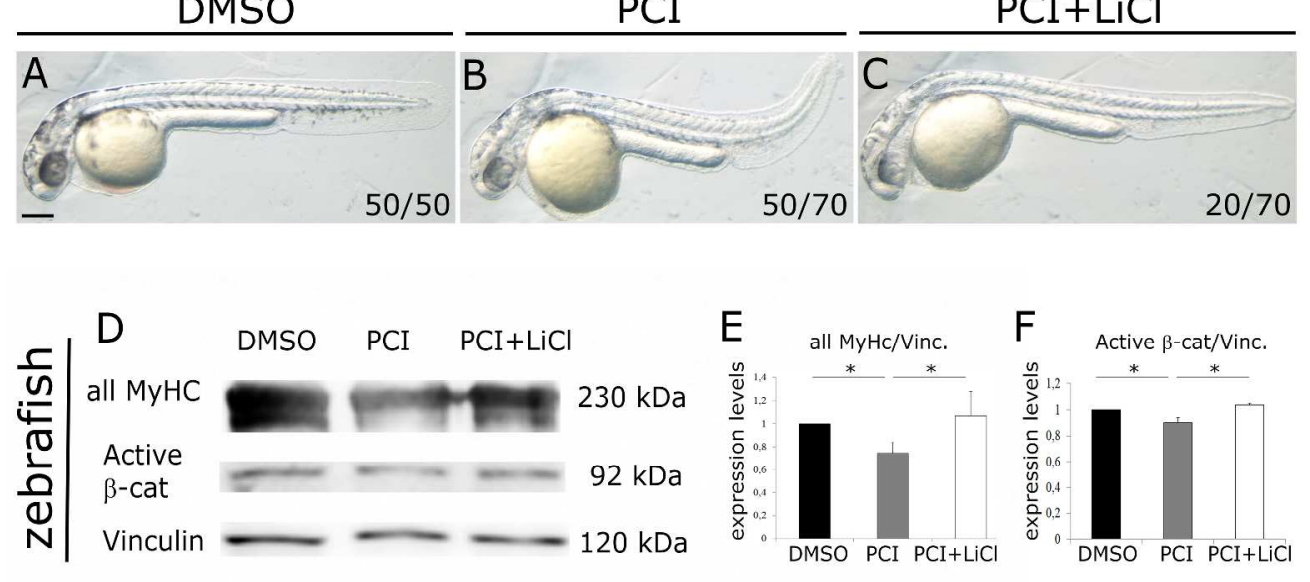

Figure 4: The HDAC8-mediated positive regulation of Wnt signalling is responsible for skeletal muscle differentiation. (A-C) Morphological defect presented by PCI-treated embryos were rescued by $\mathrm{LiCl}$ addition. (D-F) Skeletal muscle differentiation was rescued when the Wnt pathway was restored by LiCl in PCI treated zebrafish embryos. (D) Sarcomeric myosins, analysed by Western blot techniques, decreased in PCI treated embryos and returned comparable to those treated with DMSO when Wnt pathway was rescued adding LiCl (quantification in $\mathrm{E}$ ). The efficacy of $\mathrm{LiCl}$ treatment was verify measuring the active $\beta$-catenin by Western blot techniques (quantification in F). Asterisks represent $* p<0.05$, Student's t test. 